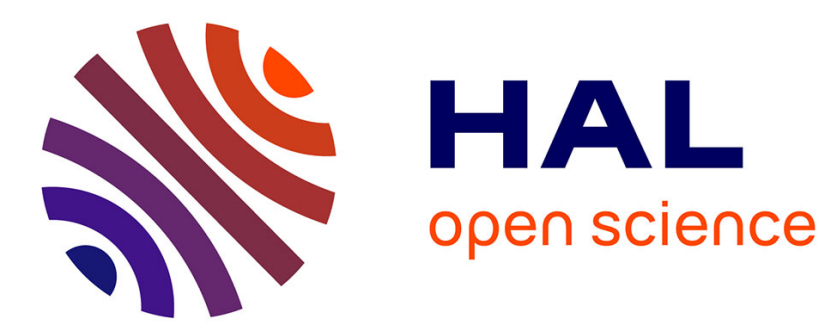

\title{
Design of robust observer for uncertain Takagi-Sugeno models
}

\author{
Abdelkader Akhenak, Mohammed Chadli, José Ragot, Didier Maquin
}

\section{To cite this version:}

Abdelkader Akhenak, Mohammed Chadli, José Ragot, Didier Maquin. Design of robust observer for uncertain Takagi-Sugeno models. IEEE International Conference on Fuzzy Systems, FUZZ-IEEE 2004, Jul 2004, Budapest, Hungary. pp.CDROM. hal-00151295

\section{HAL Id: hal-00151295 \\ https://hal.science/hal-00151295}

Submitted on 29 Mar 2014

HAL is a multi-disciplinary open access archive for the deposit and dissemination of scientific research documents, whether they are published or not. The documents may come from teaching and research institutions in France or abroad, or from public or private research centers.
L'archive ouverte pluridisciplinaire HAL, est destinée au dépôt et à la diffusion de documents scientifiques de niveau recherche, publiés ou non, émanant des établissements d'enseignement et de recherche français ou étrangers, des laboratoires publics ou privés. 


\title{
Design of Robust Observer for Uncertain Takagi-Sugeno Models
}

\author{
A. Akhenak, M. Chadli, J. Ragot, D. Maquin \\ Centre de Recherche en Automatique de Nancy, \\ INPL CNRS UMR 7039. \\ 2, Avenue de la forêt de Haye. 54516 Vandoeuvre, France. \\ E-mail: \{aakhenak, mchadli, jragot, dmaquin\}@ensem.inpl-nancy.fr
}

\begin{abstract}
This paper deals with the robust fuzzy observer design problem for a class of uncertain nonlinear system represented by Takagi-Sugeno model. Stability conditions of such observers are expressed in terms of linear matrix inequalities (LMI). An example of simulation is given to illustrate the proposed method.
\end{abstract}

\section{INTRODUCTION}

The state reconstruction of an uncertain system is a traditional problem of the automatic. The observer of Lunberger is not always sufficient for the fault detection, because the state estimation error given by this observer for an uncertain system or with unknown inputs does not converge inevitably towards zero.

In the linear system framework, observers can be designed for singular systems, unknown input systems, delay systems and also uncertain system with time-delay perturbations [4][7]. In the case of a nonlinear system affected by unknown inputs and with a multiple model representation, a technique for multiple model state estimation by using a fuzzy observer with sliding mode has been proposed [1].

In this paper, we consider the state estimation of an uncertain Takagi-Sugeno (T-S) model [5]. For that purpose a fuzzy observer based on convex interpolation of classical Luenberger observers [3] involving additive terms used to overcome the uncertainties is designed. Using quadratic Lyapunov function, sufficient asymptotic stability conditions are given in LMI formulation [2].

The following notation is used: $P>0$ denotes a symmetric positive definite matrix, and $I_{N}=\{1, \ldots, N\}$.

\section{PROBLEM Formulation}

An uncertain T-S model is represented as follows:

$$
\left\{\begin{array}{l}
\dot{x}(t)=\sum_{i=1}^{N} \mu_{i}(\xi)\left(\left(A_{i}+\Delta A_{i}(t)\right) x(t)+B_{i} u(t)\right) \\
y(t)=\sum_{i=1}^{N} \mu_{i}(\xi) C_{i} x(t)
\end{array}\right.
$$

$$
\sum_{i=1}^{N} \mu_{i}(\xi)=1, \text { with } 0 \leq \mu_{i}(\xi) \leq 1, \quad \forall i \in I_{N}
$$

where $N$ is the number of local models, $x(t) \in \mathbb{R}^{n}$ is the state vector, $u(t) \in \mathbb{R}^{m}$ is the input vector and $y(t) \in \mathbb{R}^{p}$ is the output vector. The ith local model, is characterized by the matrices $A_{i} \in \mathbb{R}^{n \times n}, B_{i} \in \mathbb{R}^{n \times m}, C_{i} \in \mathbb{R}^{p \times n}$ and the correponding activation function $\mu($.$) . Finally, \xi$ represents the vector of decision depending on the input and/or the measurable state variables. $\Delta A_{i}(t) \in \mathbb{R}^{n \times n}$ are modeling/approximation errors which have to be taken into account in the fuzzy observer design. Note that other matched and unmatched uncertainties are lumped together with these errors. The error $\Delta A_{i}$ is bouded such as:

$$
\left\|\Delta A_{i}(t)\right\|<\delta_{i}
$$

where $\|$.$\| is spectral norm.$

\section{MAIN RESUlT}

\section{A. Fuzzy Observer Design}

Assuming that all the pairs $\left(A_{i}, C_{i}\right)$ are observable, the following structure of fuzzy observer is proposed:

$$
\left\{\begin{array}{l}
\dot{\hat{x}}(t)=\sum_{i=1}^{N} \mu_{i}(\xi)\left(A_{i} \hat{x}(t)+B_{i} u(t)+H_{i}(y(t)-\hat{y}(t))+\alpha_{i}(t)\right) \\
\hat{y}(t)=\sum_{i=1}^{N} \mu_{i}(\xi) C_{i} \hat{x}(t)
\end{array}\right.
$$

where $\hat{x}(t) \in \mathbb{R}^{n}$ is the state estimation, $\hat{y}(t) \in \mathbb{R}^{p}$ is the observer output. The gain $H_{i} \in \mathbb{R}^{n \times m}$ of the ith local observer and the term $\alpha_{i}(t) \in \mathbb{R}^{n}$ might be determined to force state estimation error converging to zero. Define the state estimation error as

$$
e(t)=x(t)-\hat{x}(t)
$$

and the output residual as

$$
r(t)=y(t)-\hat{y}(t)=\sum_{i=1}^{N} \mu_{i}(\xi) C_{i} e(t)
$$


The state estimation error is governed by:

$$
\begin{gathered}
\dot{e}(t)=\dot{x}(t)-\dot{\hat{x}}(t) \\
=\sum_{i=1}^{N} \sum_{j=1}^{N} \mu_{i}(\xi) \mu_{j}(\xi)\left(\bar{A}_{i j} e(t)+\Delta A_{i}(t) x-\alpha_{i}(t)\right) \\
\bar{A}_{i j}=A_{i}-H_{i} C_{j}
\end{gathered}
$$

The following lemma is needed to prove theorem 1 .

Lemma1 : For any matrices $X$ and $Y$ with appropriate dimensions, the following property holds:

$$
X^{T} Y+Y^{T} X \leq X^{T} X+Y^{T} Y
$$

Theorem1 : Suppose that there exists symmetric matrix $P>0$ and matrices $H_{i}$ such that

$$
\bar{A}_{i j}^{T} P+P \bar{A}_{i j}+P^{2}+2 \delta_{i}^{2} I<0, \forall(i, j) \in I_{N}^{2}
$$

where $\bar{A}_{i j}$ is defined by (8), then the state estimation error of the robust state fuzzy observer (4) with condition(10) converges asymptotically to zero:

$\alpha_{i}(t)= \begin{cases}\frac{\delta_{i}^{2} \hat{x}^{T}(t) \hat{x}(t)}{r(t)^{T} r(t)} P^{-1} \sum_{i=1}^{N} \mu_{i}(\xi) C_{i}^{T} r(t), & \text { if } r(t) \neq 0 \\ 0, & \text { if } r(t)=0\end{cases}$

Proof: Consider the following Lyapunov function candidate

$$
V(t)=e(t)^{T} P e(t), \quad P>0
$$

The time derivatives of $V(t)$ along the trajectories of system (7) satisfy,

$$
\begin{aligned}
\dot{V}(t) & =\dot{e}^{T}(t) P e(t)+e^{T}(t) P \dot{e}(t) \\
& =\sum_{i=1}^{N} \sum_{j=1}^{N} \mu_{i}(\xi) \mu_{j}(\xi)\left(e^{T}(t)\left(\bar{A}_{i j}^{T} P+P \bar{A}_{i j}\right) e(t)+\right. \\
& \left.2 x^{T}(t) \Delta A_{i}^{T} P e(t)-2 \alpha_{i}^{T}(t) P e(t)\right)
\end{aligned}
$$

Using lemma (1), the time derivative of $V(t)$ can be written as:

$$
\begin{gathered}
\dot{V}(t) \leq \sum_{i=1}^{N} \sum_{j=1}^{N} \mu_{i}(\xi) \mu_{j}(\xi)\left(e^{T}(t)\left(\bar{A}_{i j}^{T} P+P \bar{A}_{i j}+P^{2}\right) e(t)\right. \\
\left.-2 \alpha_{i}^{T}(t) P e(t)+x^{T}(t) \Delta A_{i}^{T} \Delta A_{i} x(t)\right) \\
\leq \sum_{i=1}^{N} \sum_{j=1}^{N} \mu_{i}(\xi) \mu_{j}(\xi)\left(e^{T}(t)\left(\bar{A}_{i j}^{T} P+P \bar{A}_{i j}+P^{2}\right) e(t)+\right. \\
\left.\delta_{i}^{2} x^{T}(t) x(t)-2 \alpha_{i}^{T}(t) P e(t)\right)
\end{gathered}
$$

Using the expression (5) and the property of lemma 1, the derivative of the Lyapunov function becomes as follows:

$$
\begin{array}{r}
\dot{V}(t) \leq \sum_{i=1}^{N} \sum_{j=1}^{N} \mu_{i}(\xi) \mu_{j}(\xi)\left(e^{T}(t)\left(\bar{A}_{i j}^{T} P+P \bar{A}_{i j}+P^{2}\right) e(t)+\right. \\
\left.\delta_{i}^{2}(\hat{x}(t)+e(t))^{T}(\hat{x}(t)+e(t))-2 \alpha_{i}^{T}(t) P e(t)\right) \\
\leq \sum_{i=1}^{N} \sum_{j=1}^{N} \mu_{i}(\xi) \mu_{j}(\xi)\left(e ^ { T } ( t ) \left(\bar{A}_{i j}^{T} P+P \bar{A}_{i j}+P^{2}+\right.\right. \\
\left.\left.2 \delta_{i}^{2} I\right) e(t)+2 \delta_{i}^{2} \hat{x}^{T}(t) \hat{x}(t)-2 \alpha_{i}^{T}(t) P e(t)\right)
\end{array}
$$

Case 1: $r(t) \neq 0$ - with the expression (5), the inequality (13) becomes:

$$
\begin{aligned}
\alpha_{i}^{T}(t) P e(t) & =\delta_{i}^{2} \frac{\hat{x}^{T}(t) \hat{x}(t)}{r^{T}(t) r(t)} r^{T}(t) \sum_{k=1}^{N} \mu_{k}(\xi) C_{k} P^{-1} P e(t) \\
& =\delta_{i}^{2} \frac{\hat{x}^{T}(t) \hat{x}(t)}{r^{T}(t) r(t)} r^{T}(t) r(t)=\delta_{i}^{2} \hat{x}^{T}(t) \hat{x}(t)
\end{aligned}
$$

Then, the expression (13) can be rewritten as follows:

$\dot{V}(t) \leq \sum_{i=1}^{N} \sum_{j=1}^{N} \mu_{i}(\xi) \mu_{j}(\xi) e^{T}(t)\left(\bar{A}_{i j}^{T} P+P \bar{A}_{i j}+P^{2}+2 \delta_{i}^{2} I\right) e(t)$

Case 2: $r(t)=0$ - we obtain directly (14).

Consequently the conditions (9) guarantee that $\dot{V}<0$, and then the estimation error converges to zero asymptotically

The conditions (9) are nonlinear in $P$ and $H_{i}$, but they can be easily converted into a LMI problem as it will be shown at the end of the next section.

\section{B. Relaxed Stability Conditions}

In order to reduce the conservatism of the inequalities (9), we propose to design the observer thanks to theorem (2). The following lemma is used for proof by taking into account the maximum number $\mathrm{s}$ of local model simultaneously activated ( $s$ is depending on the support of the activation function).

Lemma2 : Taking into account the properties of the activation functions (2), the following inequality holds [6]:

$$
\sum_{i=1}^{N} \mu_{i}^{2}(\xi) \geq \frac{2}{s-1} \sum_{\substack{i=1 \\ i<j}}^{N} \sum_{j=1}^{N} \mu_{i}(\xi) \mu_{j}(\xi), 2 \leq s \leq N
$$

Theorem2 : Suppose that there exists symmetric matrices $P>$ $0, Q \geq 0$ and matrices $H_{i}$ such that

$$
\begin{gathered}
\bar{A}_{i i}^{T} P+P \bar{A}_{i i}+P^{2}+2 \delta_{i}^{2} I+(s-1) Q<0, \quad i \in I_{N} \\
\frac{1}{2}\left[\left(\bar{A}_{i j}+\bar{A}_{j i}\right)^{T} P+P\left(\bar{A}_{i j}+\bar{A}_{j i}\right)\right]+P^{2}+2 \delta_{i}^{2} I-Q<0 \\
\forall i<j \in I_{N}
\end{gathered}
$$

where $\bar{A}_{i j}$ is defined by (8), then the state estimation error of the robust state fuzzy observer (4) with condition (10) 
converges asymptotically to zero.

Proof : Starting from the expression (13), we can write

$$
\begin{aligned}
\dot{V}(t) & \leq \sum_{i=1}^{N} \sum_{j=1}^{N} \mu_{i}(\xi) \mu_{j}(\xi) e^{T}(t)\left(\bar{A}_{i j}^{T} P+P \bar{A}_{i j}+P^{2}+2 \delta_{i}^{2} I\right) e(t \\
= & \sum_{i=1}^{N} \mu_{i}(\xi)^{2} e^{T}(t)\left(\bar{A}_{i i}^{T} P+P \bar{A}_{i i}+P^{2}+2 \delta_{i}^{2} I\right) e(t) \\
& +2 \sum_{i=1}^{N} \sum_{\substack{j=1 \\
i<j}}^{N} \mu_{i}(\xi) \mu_{j}(\xi) e^{T}(t)\left(P^{2}+2 \delta_{i j}^{2} I+\right. \\
& \left.\left.\left.\bar{A}_{j i}\right)^{T} P+P\left(\bar{A}_{i j}+\bar{A}_{j i}\right)\right]\right) e(t)
\end{aligned}
$$

on the choice of $\varepsilon$. May simulations, not reported in this paper, have provide the efficiency of the proposed observer design.

\section{Simulation EXAmple}

tConsider the fuzzy model, made up of two local models and involving two outputs and three states.

$$
\left\{\begin{array}{l}
\dot{x}(t)=\sum_{i=1}^{2} \mu_{i}(\xi)\left(\left(A_{i}+\Delta A_{i}\right) x(t)+B_{i} u(t)\right) \\
y(t)=C x(t)
\end{array}\right.
$$

The numerical values of matrices $A_{i}, B_{i}, C_{i}$ and $R_{i}$ are as follows:

Using the expression (16), we deduce:

$\dot{V}(t) \leq e^{T}(t)\left(-\sum_{i=1}^{N} \mu_{i}^{2}(\xi)(s-1)+2 \sum_{\substack{i=1 \\ i<j}}^{N} \sum_{j=1}^{N} \mu_{i}^{2}(\xi) \mu_{j}(\xi)\right) Q e(t)$

Lemma (2) ensures that $\dot{V}<0$, then the estimation error converges asymptotically to zero.

The conditions (16) are nonlinear in $P$ and $H_{i}$. In order to convert them into a LMI problem, the following change of variables $W_{i}=P H_{i}$, gives:

$$
\begin{array}{r}
A_{i}^{T} P+P A_{i}-C_{i}^{T} W_{i}^{T}-W_{i} C_{i}+P^{2}+2 \delta_{i}^{2} I+(s-1) Q<0 \\
\frac{\left(A_{i}+A_{j}\right)^{T}}{2} P+P \frac{\left(A_{i}+A_{j}\right)}{2}-C_{j}^{T} W_{i}^{T}-W_{i} C_{j}-C_{i}^{T} W_{j}^{T}- \\
W_{j} C_{i}+P^{2}+2 \delta_{i}^{2} I-Q<0
\end{array}
$$

Applying the Schur complement [1], we obtain from (18) the following LMI formulation:

$$
\begin{aligned}
& {\left[\begin{array}{cc}
A_{i}^{T} P+P A_{i}-C_{i}^{T} W_{i}^{T}-W_{i} C_{i}+(s-1) Q+2 \delta_{i}^{2} I & P \\
P & -I
\end{array}\right]<} \\
& {\left[\begin{array}{cc}
\frac{\left(A_{i}+A_{j}\right)^{T}}{2} P+P \frac{\left(A_{i}+A_{j}\right)}{2}-C_{j}^{T} W_{i}^{T}-W_{i} C_{j}- & P \\
C_{i}^{T} W_{j}^{T}-W_{j} C_{i}-Q+2 \delta_{i}^{2} I & \\
P & -I
\end{array}\right]<0}
\end{aligned}
$$

Summarising, the state observer is fully described by (4), (6), (10), the Lyapunov matrix $P$ and the gains $H_{i}=P^{-1} W_{i}$.

It is important to note that there is potentiel problem in implementation of this observer : when the state estimation error $e(t)$ goes to zero, the magnitude $\alpha_{i}(t)$ may increase without bound. This problem is overcome as follows. When $\left|e_{i}(t)\right|<$ $\varepsilon$, where $\varepsilon$ is a small positif number and $e(t)=\left(e_{1}, e_{2}, \ldots, e_{n}\right)$, we set $e_{i}=\varepsilon$ and the denominator of (10) becomes $r^{T} r=$ $\sum_{i=1}^{N} \sum_{j=1}^{N} \mu_{i}(\xi) \mu_{j}(\xi) \nu_{i j} \varepsilon^{2}$ with $\nu_{i j}$, positive scalar, is depending on $C_{i}$. In this case, the estimation error cannot converge to zero asymptotically but to a small neighborhood of zero depending

$$
\begin{aligned}
& A_{1}=\left[\begin{array}{ccc}
-2 & 1 & 1 \\
1 & -3 & 0 \\
2 & 1 & -6
\end{array}\right] \quad A_{2}=\left[\begin{array}{ccc}
-3 & 2 & 2 \\
5 & -8 & 0 \\
0.5 & 0.5 & -4
\end{array}\right] \\
& B_{1}=\left[\begin{array}{c}
1 \\
0.5 \\
0.5
\end{array}\right] \quad B_{2}=\left[\begin{array}{c}
0.5 \\
1 \\
0.25
\end{array}\right] \quad C=\left[\begin{array}{lll}
1 & 1 & 1 \\
1 & 0 & 1
\end{array}\right]
\end{aligned}
$$

Model of uncertainties are such that $\Delta A_{i}(j, k)(t)=$ $0.1 A_{i}(j, k) \eta(t)(j, k) \in\{1,3\}$, the function $\eta(t)$ is a Gaussian random function with zero mean and a unity variance.

In this case, the multiple observer that estimates the state vector of the multiple model is described by,

$\left\{\begin{array}{l}\dot{\hat{x}}(t)=\sum_{i=1}^{2} \mu_{i}(\xi)\left(A_{i} \hat{x}(t)+B_{i} u(t)+G_{i}(y(t)-C \hat{x}(t))+\alpha_{i}(t)\right) \\ \hat{y}(t)=C \hat{x}(t)\end{array}\right.$

with

$$
\left(A_{i}-H_{i} C\right)^{T} P+P\left(A_{i}-H_{i} C\right)+P^{2}+2 \delta_{i}^{2} I<0, \forall i \in I_{2}
$$

It is important to note that a potential problem arises in the implementation of this multiple observer: when the output estimation error $r(t)$ tends towards zero, the magnitude of $\alpha_{i}(t)$ may increase without bound. This problem is overcome as follows.

The terms $\alpha_{i}(t)$ are fixed to zero when the output estimation error us such that $\|r(t)\|<\varepsilon$ a small positive number chosen by the user. In this case, the estimation error cannot converge to zero asymptotically but to a small neighborhood of zero depending on the choice of $\varepsilon$.

\section{A. Simulation Results}

The resolution of equations (21) using LMI tools leads to the following matrices $H_{i}$ and $P$ :

$$
P=\left(\begin{array}{ccc}
0.6365 & -0.5836 & 0.0332 \\
-0.5836 & 0.9584 & -0.1814 \\
0.0332 & -0.1814 & 0.2343
\end{array}\right)
$$




$$
H_{1}=\left(\begin{array}{cc}
-0.26 & 7.34 \\
28.97 & -29.28 \\
0.64 & 7.30
\end{array}\right) \quad H_{2}=\left(\begin{array}{cc}
7.00 & 4.55 \\
38.82 & -33.34 \\
1.11 & 8.50
\end{array}\right)
$$

The simulation results are represented on the following figures. The convergence of the state vector of the fuzzy observer towards those of the fuzzy model is quite good. At the vicinity of $t=0$, the disparity between estimated and actual state is due to the choice of initial conditions.

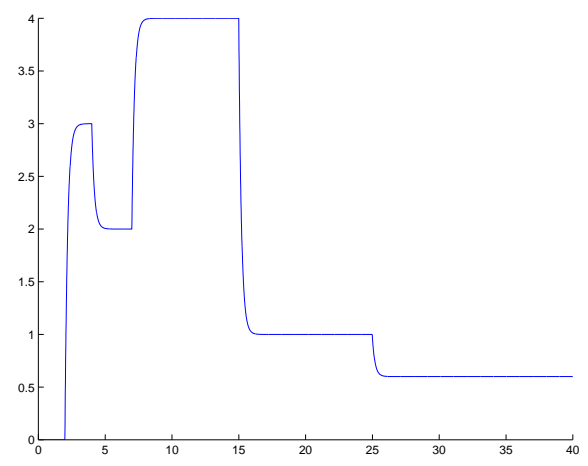

Fig. 1. The known input $u(t)$

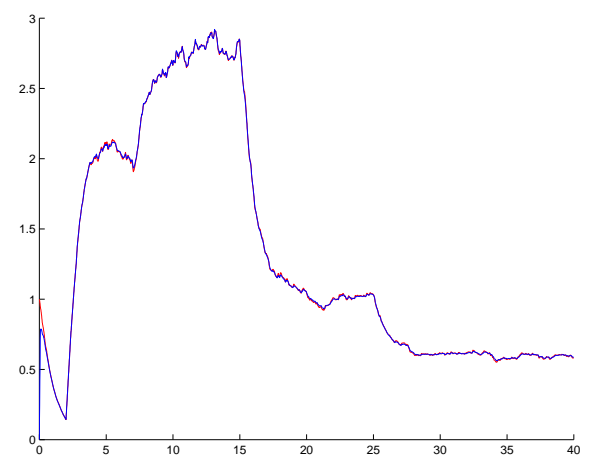

Fig. 2. The state $x_{1}(t)$ and its estimate

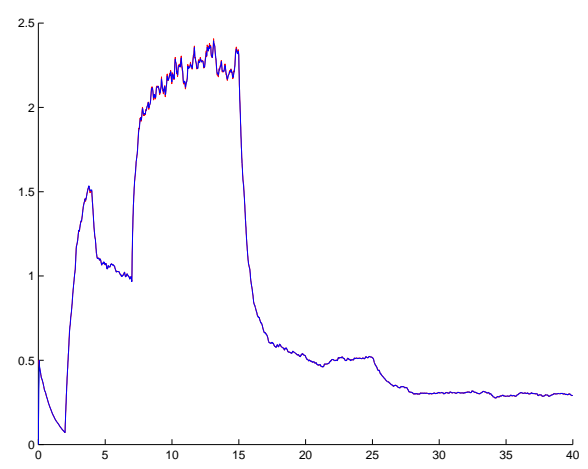

Fig. 3. The state $x_{2}(t)$ and its estimate

\section{CONCLUSION}

A robust fuzzy observer design for a T-S model has been proposed in this paper. The design of such observer relies

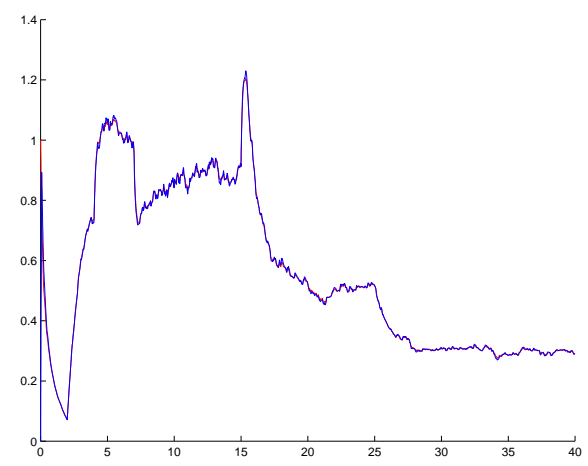

Fig. 4. The state $x_{3}(t)$ and its estimate

on the existence of a quadratic Lyapunov function ensuring asymptotic convergence of the fuzzy observer. The stability of the fuzzy observer requires however the consideration of coupling constraints between these local observers; these contraints lead to the resolution of a LMI problem under structural constraints. Assuming the existence of suited matrices, we showed that the reconstruction of the state vector of the fuzzy model is possible. The simulation results show that the estimation state is very satisfactory.

\section{REFERENCES}

[1] A. Akhenak, M. Chadli, D. Maquin and J. Ragot, Sliding mode multiple observer for fault detection and isolation, 42th IEEE CDC, Hawaii, December 9-12, 2003.

[2] S. Boyd, L. El Ghaoui, E. Feron and V. Balakrishnan, Linear Matrix Inequalities in System and Control Theory, Philadelphia: SIAM, 1994.

[3] M. Chadli, D. Maquin, J. Ragot, Output stabilization in multiple model approach, IEEE Control Conference on Application CCA'02, September 2002.

[4] K. K. Fan, J. G. Hsieh, LMI Approach to design of robust state observer for uncertain systems with time-delay perturbation, IEEE International Conference on Industrial Technology 2002, Bangkok, Thailand, pp.1111$1115,2002$.

[5] M. Takagi and M. Sugeno, Fuzzy identification of systems and its application to moddeling and control, IEEE Trans on Systems Man and Cybernetics-part C, vol. 15, n. 1, pp. 116-132, 1985.

[6] K. Tanaka, T. Ikeda and Y. Y. He, Fuzzy regulators and fuzzy observers: relaxed stability conditions and LMI-based design, IEEE Trans Fuzzy Systems, vol. 6, n. 1, pp. 250-256, May 1998.

[7] Y. Xia, Y. Jia, Robust sliding mode control for uncertain time-delay systems: an LMI approach, IEEE Trans on Automatic Control, vol. 48, n. 6, June 1998. 